\title{
An Algorithm for Initial Fluxes of Metabolic P Systems
}

\author{
Roberto Pagliarini, Giuditta Franco, Vincenzo Manca
}

University of Verona, Italy

Computer Science Department

Strada Le Grazie 15, 37134 Verona, Italy

E-mail: \{roberto.pagliarini, giuditta.franco, vincenzo.manca\}@ univr.it

Received: April 5, 2009

Accepted: May 30, 2009

\begin{abstract}
A central issue in systems biology is the study of efficient methods inferring fluxes of biological reactions by starting from experimental data. Among the different techniques proposed in the last years, the theory of Metabolic P systems, which is based on the Log-Gain principle, proved to be helpful for deducing biological fluxes from temporal series of observed dynamics. According to this approach, the algebraic systems provided by the Log-Gain principle determine the reaction fluxes underlying a system dynamics when initial fluxes are known. Here we propose a heuristic algorithm for estimating the initial fluxes, that is tested in two case studies.
\end{abstract}

Keywords: Biological modeling, P systems, MP systems, Metabolic flux estimation, Heuristic algorithms.

\section{Introduction}

In the last years, the problem of reverse-engineering of biological phenomena from experimental data has spurred increasing interest in scientific communities. For these reasons, many computational models inspired from biology have been proposed. Among these models, the Metabolic P systems [11, 12], shortly MP systems, proved to be relevant in the analysis of dynamics of biochemical processes, that is, structures where matter of different type is transformed by reactions. By means of MP systems models of several interesting phenomena were provided, among which we mention: the Lotka-Volterra dynamics $[2,3,15]$, a Susceptible-Infected-Recovered epidemic [2], the Leukocyte Selective Recruitment in the immune response [2], the Protein Kinase C Activation [3], the Mitotic Cycle [14], the Pseudomonas Quorum Sensing [4] and the Non-Photochemical Quenching phenomenon [16].

The importance of MP systems is their potential applicability to the reverse-engineering problem of biological phenomena. In fact, in the framework of MP systems, a theory called Log-Gain [10, 11, 12] has been introduced, specifically devoted to the deduction of reaction fluxes, that is, the amount of reactants transformed by the reactions at any step of the system.

As we will show, a key point for achieving this task consists in the discovery of the fluxes associated to the passage of a metabolic system from the state at the initial observation instant to the next one. In this paper a heuristic algorithm is proposed for estimating the initial fluxes vector from few steps of observation. In few words, the algorithm first roughly computes the initial fluxes by assuming they have a form recalling the mass action principle, and then solves a system of equations to deduce the corresponding fluxes at the next step. From these values, the algorithm evaluates how much of each substance is necessary to activate the first evolution step, and finally the actual initial fluxes are computed by solving a minimization problem.

The present paper is organized as follows. Section 2 is devoted to the definition of Metabolic P Systems, while in Section 3 Log-Gain theory is briefly recalled. In Section 4 we describe the algorithm 
which solves the initial fluxes problem. Section 5 reports the simulations of a couple of systems obtained by starting with initial fluxes computed by our algorithm. Further remarks and some directions for future research are discussed in the last Section.

\section{Metabolic P systems}

MP systems are a special class of dynamical systems (the reader can find some details concerning dynamical aspects of MP systems in [13]), based on P systems [5, 18, 19], which are related to metabolic processes. MP systems are essentially constituted by multiset grammars where rules are regulated by specific functions depending on the state of the system. From a membrane computing point of view, MP systems can be seen as deterministic mono-membrane P systems where the transitions between states are calculated by a suitable recurrent equation. In an MP system the variation of the whole system is considered in a macroscopic time interval. In this manner, the evolution law of the system includes the knowledge of the contribution of each reaction in the evolution from one state to the next one. Therefore, dynamics is given at discrete steps, and in each step, it is ruled by a partition of matter among the reactions transforming it. The principle underlying the partitioning is called mass partition principle, and it defines the transformations of object populations, rather than single objects, according to a suitable generalization of chemical laws [11].

The following definition introduces the MP systems in a formal way ( $\mathbb{N}, \mathbb{Z}$, and $\mathbb{R}$ denote the sets of natural, integer, and real numbers, respectively).

Definition 1 (MP system). An MP system $M$ is specified by the following construct:

$$
M=(X, R, V, H, \Phi, \nu, \mu, \tau)
$$

where $X, R$ and $V$ are finite disjoint sets, and moreover the following conditions hold, with $n, m, k \in \mathbb{N}$ :

- $X=\left\{x_{1}, x_{2}, \ldots, x_{n}\right\}$ is a finite set of substances. This set represents the types of molecules;

- $R=\left\{r_{1}, r_{2}, \ldots, r_{m}\right\}$ is a finite set of reactions. A reaction $r$ is a pair of type $\alpha_{r} \rightarrow \beta_{r}$, where $\alpha_{r}$ identifies the multiset of the reactants (substrates) of $r$ and $\beta_{r}$ identifies the multiset of the products of $r$ ( $\lambda$ represents the empty multiset). The stoichiometric matrix $\mathbb{A}$ of a set $R$ of reactions over a set $X$ of substances is $\mathbb{A}=\left(\mathbb{A}_{x, r} \mid x \in X, r \in R\right)$ with $\mathbb{A}_{x, r}=\left|\beta_{r}\right|_{x}-\left|\alpha_{r}\right|_{x}$, where $\left|\alpha_{r}\right|_{x}$ and $\left|\beta_{r}\right|_{x}$ respectively denote the number of occurrences of $x$ in $\alpha_{r}$ and $\beta_{r}$. Of course, a reaction $r$ can be seen as the vector $r=\left(\mathbb{A}_{x, r} \mid x \in X\right)$ of $\mathbb{R}^{n}$. We also set $R_{\alpha}(x)=\left\{r \in R \mid x \in \alpha_{r}\right\}, R_{\beta}(x)=\left\{r \in R \mid x \in \beta_{r}\right\}$, and $R(x)=R_{\alpha}(x) \cup R_{\beta}(x)$;

- $V=\left\{v_{1}, v_{2}, \ldots, v_{k}\right\}$ is a finite set of parameters. This set represents entities which affect the dynamics but are not transformed by reactions;

- $H=\left\{h_{v} \mid v \in V\right\}$ is a set of parameters evolution functions. The function $h_{v}: \mathbb{N} \rightarrow \mathbb{R}$ states the value of parameter $v$, and $H[i]=\left(h_{v}(i) \mid v \in V\right)$;

- $\Phi=\left\{\varphi_{r} \mid r \in R\right\}$ is the set of flux regulation maps, where, for each $r \in R, \varphi_{r}: \mathbb{R}^{n+k} \rightarrow \mathbb{R}$. Let $q \in \mathbb{R}^{n}$ be the vector of substance values and $s \in \mathbb{R}^{k}$ be the vector of parameter values. Then $(q, s) \in \mathbb{R}^{n+k}$ is the state of the system. We set by $U(q, s)=\left(\varphi_{r}(q, s) \mid r \in R\right)$ the flux vector in the state $(q, s)$, constituted by the state $q$ and by the parameters state $s$;

- $v$ is a natural number which specifies the number of molecules of a (conventional) mole of $M$;

- $\mu$ is a function which assigns, to each $x \in X$, the mass $\mu(x)$ of a mole of $\mathrm{x}$ (with respect to some measure units). 
- $\tau$ is the temporal interval between two consecutive observation steps;

Let $X[i]=\left(x_{1}[i], x_{2}[i], \ldots, x_{n}[i]\right)$, for each $i \in \mathbb{N}$, be the vector of substances values at the step $i$, and let $X[\mathrm{o}]$ be the initial values of substances. The dynamics of an MP system is completely identified by the following recurrent equation, called Equational Metabolic Algorithm, shortly EMA:

$$
X[i+1]=\mathbb{A} \times U(X[i], H[i])+X[i]
$$

where $\mathbb{A}$ is the stoichiometric matrix of reactions having dimension $n \times m$, while $\times,+$, are the usual matrix product and vector sum. We denote by $E M A[i]$ the system (1), which allows us to obtain the vector $X[i+1]$ from vectors $X[i]$ and $U(X[i], X[i])$.

If in an MP system the elements $v, \mu$, and $\tau$ are omitted, then the result is called MP grammar. It is a multiset rewrite grammar where rules are regulated by specific functions. Such a grammar is completely specified by: $i$ ) reactions, ii) flux regulation functions, iii) parameter evolution functions, $i v$ ) substances, which are the elements occurring in the reactions, and their initial values and $v$ ) parameters, which are the arguments of flux regulation functions different from substances. Parameter evolution maps and/or initial values of substances may be omitted when only the MP grammar structure is specified.

\section{Log-Gain Theory: a brief recall}

The starting point of the Log-Gain theory [10, 11, 12] for MP systems is the Allometry Law [1, 7], which has many possible formulations [10], but, in the case here discussed, it can be expressed in a simple way. Namely, a proportion can be assumed, at each step, between the relative variations of the flux of a reaction and the sum of relative variations of its reactants, with a possible gap, called offset.

Given the dynamics of an MP system, we will use the following simplified notations, for $i \in \mathbb{N}$, and $r \in R:$

$$
u_{r}[i]=\varphi_{r}(X[i], H[i]) \quad \text { and } \quad U[i]=\left(u_{r}[i] \mid r \in R\right) .
$$

Assuming to know the vectors $X[i]$ and $X[i+1]$, the equation (1) can be rewritten in the following form, which we call $A D A[i]$ (Avogadro and Dalton Action [12]):

$$
X[i+1]-X[i]=\mathbb{A} \times U[i] .
$$

Formula (3) expresses a system of $n$ equations and $m$ variables ( $n$ is the number of substances and $m$ the number of reactions) which is assumed to have maximal rank. This assumption is not restrictive. In fact, if it does not hold, the rows which are linearly dependent on other rows can be removed, by keeping the notations $\mathbb{A}, X[i+1]$ and $X[i]$ for the stoichiometric matrix and the vectors of concentration of substances, respectively. We assume thus that $\mathbb{A}$ has maximum rank, which we newly call $n$. Then there exist $n$ linearly independent reactions of $R$, and we call $R_{0}$ such a subset of reactions. From a metabolic point of view, this means that fluxes of each reaction of $R$ can be obtained as linear combination of fluxes of the reactions of $R_{0}$.

Formally, $A D A[i]$ is essentially the system $E M A[i]$ introduced in Section 2. However, these two systems have dual interpretations. In fact, in $E M A[i]$, the vectors $U[i]$ and $X[i]$ are known, and the vector $X[i+1]$ is computed by means of them, while in $A D A[i]$, the vector $X[i+1]-X[i]$ is known and $U[i]$ is computed by solving a system comprised of both the equations in $A D A[i]$ and further equations, dictated by the following Log-Gain principle, to state the reaction regulation level, as we will see by formula (6).

Indeed, since the number of reactions is realistically assumed greater than the number of substances, then system (3) has more than one solution. Therefore, fluxes cannot be univocally deduced by means of $A D A[i]$. The Log-Gain principle allows us to add more equations in order to get a univocally solvable system which could provide the flux vector. 
The two following definitions state the Log-Gain principle. For the detailed motivations of this principle we refer to papers on MP systems theory $[10,11,12]$. Further developments providing theoretical and experimental evidences of this principle will be matter of forthcoming papers.

Definition 2 (Discrete Log-Gain). Let $(z[i] \mid i \in \mathbb{N})$ be a real valued sequence. Then, the discrete log-gain of $z$, for each step $i$, is given by the following equation:

$$
\operatorname{Lg}(z[i])=\frac{z[i+1]-z[i]}{z[i]} .
$$

Principle 1 (Log-Gain regulation). Let $U[i]$ be the vector of fluxes at step $i$, for $i \geq 0$, and let $R_{0} \subset R$ be a set of $n$ linearly independent vectors of $\mathbb{R}^{n}$. Then, the Log-Gain regulation can be expressed in terms of matrix and vector operations:

$$
(U[i+1]-U[i]) / U[i]=\mathbb{B} \times \operatorname{Lg}(X[i])+C \otimes P[i+1]
$$

where:

- $\mathbb{B}=\left(p_{r, x} \mid r \in R, x \in X\right)$ where $p_{r, x} \in\{0,1\}$ with $p_{r, x}=1$ if $x$ is a reactant of $r$ and $p_{r, x}=0$ otherwise;

- $\operatorname{Lg}(X[i])=(\operatorname{Lg}(x[i]) \mid x \in X)$ is the column vector of log-gains of substances;

- $C=\left(c_{r} \mid r \in R\right)$, where $c_{r}=1$ if $r \in R_{0}$, while $c_{r}=0$;

- $P[i+1]$ is a column vector of values associated with the reactions and called (Log-Gain) offsets at step $i+1$;

- $\times$ denotes the usual matrix product;

-,,$+- /, \otimes$ denote the component-wise sum, subtraction, division and product of vectors.

If we assume to know the flux unit vector at step $i$ and put together the equations (5) and (3) at steps $i$ and $i+1$ respectively, we get the following linear system called Offset Log-Gain Adjustment module at step $i$, shortly $O L G A[i]$, where the number of variables (reported in bold font) is equal to the number of equations:

$$
\begin{aligned}
\mathbb{A} \times \boldsymbol{U}[\boldsymbol{i}+\mathbf{1}] & =X[i+2]-X[i+1] \\
(\boldsymbol{U}[\boldsymbol{i}+\mathbf{1}]-U[i]) / U[i] & =\mathbb{B} \times \operatorname{Lg}(X[i])+\boldsymbol{C} \otimes \boldsymbol{P}[\boldsymbol{i}+\mathbf{1}] .
\end{aligned}
$$

Given the vector $\operatorname{Lg}(X[i])$, for $i=0,1, \ldots, l$, where $l \in \mathbb{N}$, it is possible to prove that $O L G A[i]$, for $i=0,1, \ldots, l-1$, univocally provides $U[i]$ for $i=1,2, \ldots, l-1$.

\section{An algorithm to estimate initial metabolic fluxes}

The iteration of the OLGA module, introduced in the previous section in order to deduce the fluxes of reactions, assumes the knowledge of the initial values of fluxes. This leads to the formulation of the following problem.

Problem 1 (Initial Fluxes Problem). Given $X[0]$ and $X[1]$, find a flux vector $U[0]$ such that it satisfies the initial dynamics, that is:

$$
X[1] \approx \mathbb{A} \times U[0]+X[0]
$$

where $\approx$ means that we are searching for the vector $U[0]$ providing the minimum value of the stoichiometric error, defined as $(\|\cdot\|$ represents the Euclidean norm)

$$
\|\mathbb{A} \times U[0]-(X[1]-X[0])\| .
$$

The algorithm given below solves the Initial Fluxes Problem by using the knowledge about the dynamics in the first evolution steps in order to evaluate the amount of each substance which is necessary to activate the first evolution step. 


\subsection{The proposed algorithm}

Our algorithm consists of three phases, some of which include different computational steps. The first phase consists in the approximation of initial fluxes by assuming that fluxes are proportional to the reactant quantities product. In the second phase an OLGA module is employed to approximate the amount of substances which needs as a fuel for the first evolution step. In the third phase an optimization problem is solved, which is based on the ADA system (3). The details of the algorithm work-flow are described in the following.

Phase 1. The goal here is to roughly evaluate the initial reaction fluxes by assuming that they are proportional to the reactants for certain initial evolution steps $i$. This could appear restrictive, but at this stage we require only an initial approximation. Therefore, at a given step $i$, for all $r \in R$, we set:

$$
\widehat{u}_{r}[i]=k_{r} y_{r}[i]
$$

where $k_{r} \in \mathbb{R}$, and $y_{r}[i]$ is the product of all substance quantities, at the step $i$, which are reactants for $r$. We suppose that if $\alpha_{r}=\lambda$ then $y_{r}[i]=1$, and we set

$$
\hat{U}[i]=\left(\hat{u}_{r}[i] \mid r \in R\right)
$$

For example, in a metabolic system having three kinds of substances, $a, b, c$, and as a set of reactions those given in the first column of Table 1, the relationships between the fluxes of these reactions and their reactants are reported in the second column of Table 1.

For any $x \in X$, let us consider the following system, called Local-Stoichiometric Module at the step $i$, where $\mathbb{A}$ is the stoichiometric matrix:

$$
x[i+1]-x[i]=\sum_{r \in R(x)} \mathbb{A}_{x, r} \widehat{u}_{r}[i]
$$

If we assume that the constants $k_{r}$, with $r \in R$, do not sensibly change in few steps, then by applying the system (9), in at most $m-n$ steps either we obtain a square linear system of dimension $m$ having maximum rank or the algorithm ends without an output. In fact, under the assumption that the rank of Local-Stoichiometric Module is $n$ (that is, the number of equations) and that the number of variables is $m$, with $n<m$, then the system is completely determined if we add other $m-n$ equations. Assuming to gain at least one new significant equation at each step $i$, then in at most $m-n$ steps we obtain a system of $(m-n) n+n$ equations with $m$ variables and rank equals to $m$. In this way, we can obtain a square linear system having unique solution.

In the example reported in Table 1, we have a Local-Stoichiometric Module of 3 equations having rank 3 which initially has 4 variables. At the second iteration of this module, starting from the step o, we

\begin{tabular}{|l|c|}
\hline Reactions & Maps \\
\hline$r_{1}: a \rightarrow b c$ & $k_{r_{1}} a$ \\
$r_{2}: b \rightarrow a$ & $k_{r_{2}} b$ \\
$r_{3}: c \rightarrow a b$ & $k_{r_{3}} c$ \\
$r_{4}: c \rightarrow c c$ & $k_{r_{4}} c$ \\
\hline
\end{tabular}

Table 1: Reactions and corresponding flux regulation maps of the Local-Stoichiometric Module. 
get other 3 equations finally giving the following system:

$$
\begin{aligned}
\boldsymbol{a}[\mathbf{1}]-\boldsymbol{a}[\mathbf{0}] & =-\boldsymbol{k}_{1} \boldsymbol{a}[\mathbf{0}]+\boldsymbol{k}_{2} \boldsymbol{b}[\mathbf{0}]+\boldsymbol{k}_{3} \boldsymbol{c}[\mathbf{0}] \\
b[1]-b[0] & =k_{1} a[0]-k_{2} b[0]+k_{3} c[0] \\
\boldsymbol{c}[\mathbf{1}]-\boldsymbol{c}[\mathbf{0}] & =\boldsymbol{k}_{1} \boldsymbol{a}[\mathbf{0}]-\boldsymbol{k}_{3} \boldsymbol{c}[\mathbf{0}]+\boldsymbol{k}_{4} \boldsymbol{c}[\mathbf{0}] \\
a[2]-a[1] & =-k_{1} a[1]+k_{2} b[1]+k_{3} c[1] \\
\boldsymbol{b}[\mathbf{2}]-\boldsymbol{b}[\mathbf{1}] & =\boldsymbol{k}_{1} \boldsymbol{a}[\mathbf{1}]-\boldsymbol{k}_{2} \boldsymbol{b}[\mathbf{1}]+\boldsymbol{k}_{3} \boldsymbol{c}[\mathbf{1}] \\
\boldsymbol{c}[\mathbf{2}]-\boldsymbol{c}[\mathbf{1}] & =\boldsymbol{k}_{1} \boldsymbol{a}[\mathbf{1}]-\boldsymbol{k}_{3} \boldsymbol{c}[\mathbf{1}]+\boldsymbol{k}_{4} \boldsymbol{c}[\mathbf{1}]
\end{aligned}
$$

where $a[0]=100, b[0]=100, c[0]=1, a[1]=101.87, b[1]=96.17$, and $c[1]=2.85$. This system has rank 4 , and 4 linearly independent equations are reported in bold font. Thus, we can obtain a system of equations having unique solution. In general, if we start with the Local-Stoichiometric Module at the step o then we can compute the vector $\hat{U}[0]=\left(\hat{u}_{r}[0] \mid r \in R\right)$ by applying the Local-Stoichiometric module for a suitable number of steps. The algorithm stops with no output if after $m-n$ iterations of the above technique, the number of equations linearly independent is less than $m$.

Phase 2. The aim of this step is to estimate the amount of substance necessary to start the first system evolution step. We describe this step along with two sub-phases.

In the first sub-phase we solve $O L G A[\mathrm{o}]$ module, with $U[\mathrm{o}]=\hat{U}[\mathrm{o}]$, where $\hat{U}[\mathrm{o}]$ is the vector of fluxes computed in the previous step. Let us call $U^{*}=\left(u_{r}^{*} \mid r \in R\right)$ the solution of this system. However, if some elements of this vector have a negative value, then we choose a different set of $n$ linearly independent reactions in $O L G A$ and newly apply the above procedure. The algorithm stops with no answer if a positive solution is not found after a number of attempts equal to the number of such different sets. However, general methods are under investigation which systematically and efficiently search for an unique positive solution $U^{*}$.

In the second sub-phase we compute, for each $x \in X$, the amount of substance $\bar{x}$ occurring for the application of the reactions in the first evolution step. If $\mathbb{A}^{-}$is the activation matrix defined by $\mathbb{A}_{x, r}^{-}=$ $\left|\alpha_{r}\right|_{x}$, for $x \in X, r \in R$, then the searched values are obtained by computing the vector $\bar{X}=\mathbb{A}^{-} \times U^{*}$.

Phase 3. In the last step we obtain the actual vector of fluxes $U^{\circ}$ by solving a norm minimization problem [9] such that $U^{\circ}$ provides the minimum of the following (Euclidean) norm

$$
\|\mathbb{A} \times \xi-(X[2]-X[1])\|
$$

over all the positive vectors $\xi=\left(\xi_{r} \mid r \in R\right)$ of $\mathbb{R}^{m}$ such that

$$
\mathbb{A}^{-} \times \xi=\bar{X}
$$

where $\bar{X}$ is the vector computed at the previous step.

\section{Experiments}

In this section, in order to evaluate the performance of our algorithm, we apply it to two case studies: i) a synthetic oscillatory metabolic system and $i i)$ the Belousov-Zhabotinsky reaction $[8,20,21]$.

\subsection{A synthetic metabolic system}

Let us consider the synthetic non-cooperative metabolic system without parameters called Sirius [11] and given by Table 2 . Firstly, we compute $U[1]=\left(\varphi_{1}\left(X[1], \varphi_{2}(X[2]), \ldots, \varphi_{5}(X[1])\right.\right.$. Then, we use our algorithm to approximate the vector of fluxes $U^{\circ}$. The two vectors are essentially the same. 


\begin{tabular}{|c|c|c|}
\hline Reactions & & Flux regulation maps \\
\hline$r_{1}: a \rightarrow a a$ & & $\varphi_{1}=k_{1} a /\left(k_{1}+k_{2} c+k_{4} b+k_{6}\right)$ \\
\hline$r_{2}: a \rightarrow b$ & & $\varphi_{2}=k_{2} a c /\left(k_{1}+k_{2} c+k_{4} b+k_{6}\right)$ \\
\hline$r_{3}: b \rightarrow \lambda$ & & $\varphi_{3}=k_{3} b /\left(k_{3}+k_{6}\right)$ \\
\hline$r_{4}: a \rightarrow c$ & & $\varphi_{4}=k_{4} a b /\left(k_{1}+k_{2} c+k_{4} b+k_{6}\right)$ \\
\hline$r_{5}: c \rightarrow \lambda$ & & $\varphi_{5}=k_{5} c /\left(k_{5}+k_{6}\right)$ \\
\hline$X[\mathrm{o}]=(100$ & $100 \quad 1)$ & $k_{1}=k_{3}=k_{5}=4, k_{2}=k_{4}=0.02, k_{6}=100$ \\
\hline
\end{tabular}

Table 2: The Sirius MP grammar.

\subsection{A biochemical case study}

In this subsection the application of the algorithm to approximate the initial fluxes of the BelousovZhabotinsky reaction, also known as BZ reaction, is discussed. This system represents a well-known example of biochemical oscillatory phenomenon, in fact this is the first evidence of a chemical clock. Although the stoichiometry of the BZ reaction is quite complicated, several simplified mathematical models of this phenomenon have been proposed. In particular, Prigogine and Nicolis [17] proposed a simplified formulation of the dynamics of the BZ reaction, called Brusselator, whose oscillating behaviour is represented by only two substances, $x$ and $y$ respectively, and it is governed by the following system of differential equations:

$$
\begin{aligned}
& \frac{d x}{d t}=k_{1}-k_{2} x+k_{3} x^{2} y-k_{4} x \\
& \frac{d y}{d t}=k_{2} x-k_{3} x^{2} y
\end{aligned}
$$

where $k_{1}=100, k_{2}={ }_{3}, k_{3}=10^{-4}$ and $k_{4}=1$ represent constant rates. We use the oscillatory dynamics obtained by solving the system (12), with initial conditions $x=1$ and $y=10$, as experimental data on which testing our algorithm. MP formulation of the Brusselator is expressed by the set of rewriting rules reported in Table 3, where, according to the literature, the fluxes of each rule $r$ depend on the concentrations of the reactants of $r$. In fact, species $x$ has two positive and two negative contributions, while one positive and one negative contributions characterize $y$. Thus, the equations can be mapped into suitable stoichiometry by following the strategy described in [6].

$$
\begin{array}{|l|}
\hline \text { Rules } \\
\hline r_{1}: \lambda \rightarrow x \\
r_{2}: x \rightarrow y \\
r_{3}: x x y \rightarrow x x x \\
r_{4}: x \rightarrow \lambda \\
\hline
\end{array}
$$

Table 3: A set of rewriting rules that describes the Brusselator stoichiometry.

In the case of BZ we adopt a different strategy of validation of our algorithm. In fact, there is a complete correspondence between the dynamics computed by the differential model and that one computed by the equational metabolic algorithm using the fluxes deduced by OLGA module (Figure 1), starting from the initial fluxes inferred by means of our algorithm.

\section{Conclusions}

The study of efficient methods for defining MP systems from experimental data is of crucial importance for systematic applications of MP systems to complex dynamics. An essential component of 

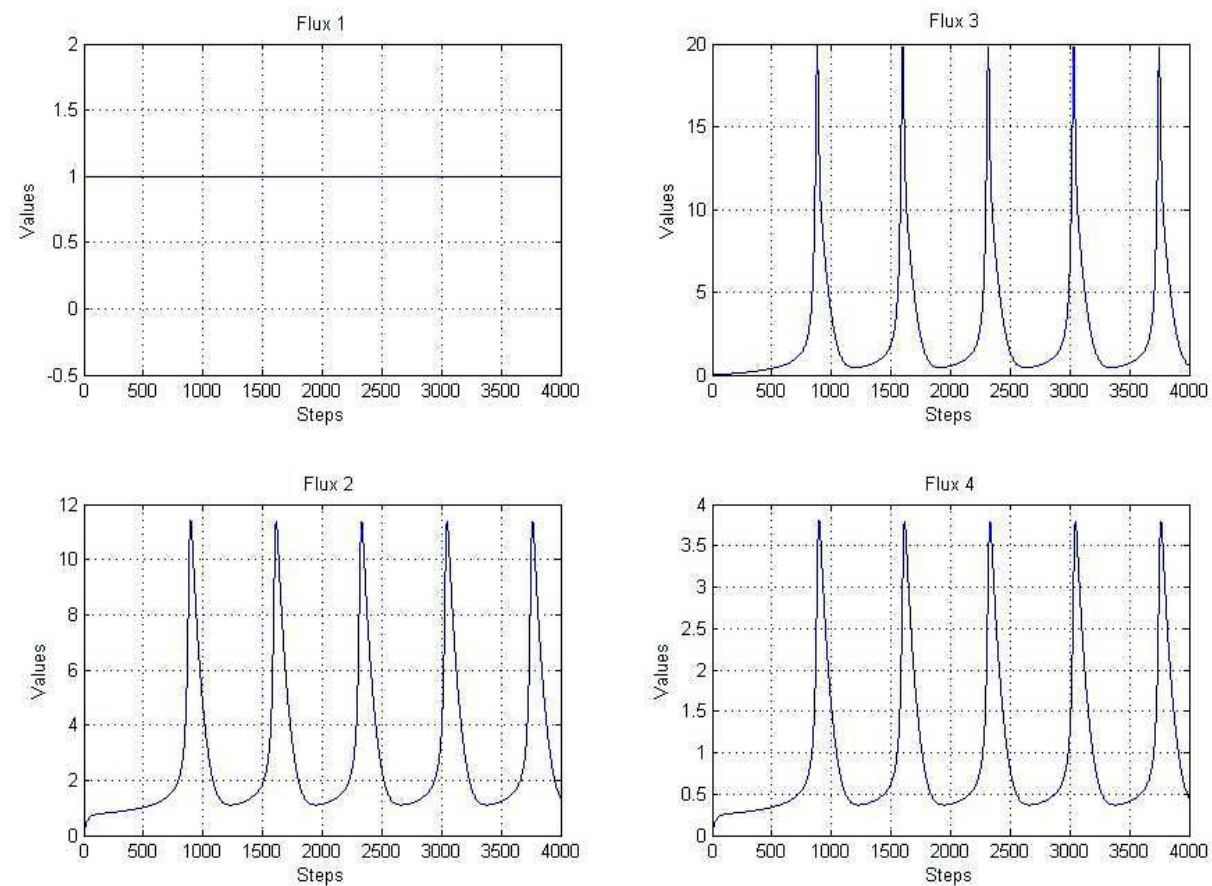

Figure 1: The BZ reaction fluxes calculated by solving the system (6) with initial vector of fluxes inferred by our algorithm.

the regulation level of an MP system can be deduced by applying the Log-Gain theory to data that can be collected from observations of the system. A crucial task to perform in this context is the reliable determination of the initial vector of fluxes.

In this paper we have devised an algorithm to infer the initial reaction fluxes of a biological network. The proposed algorithm has been validated on test cases of a synthetic metabolic oscillator and the Brusselator phenomenon. Future investigations will be developed with the aim $i$ ) to develop the computational features of this algorithm and ii) to show the applicability of our method to complex biological cases.

\section{Bibliography}

[1] L. von Bertalanffy. General Systems Theory: Foundations, Developments, Applications. George Braziller Inc, New York, NY, 1967.

[2] L. Bianco, F. Fontana, G. Franco, and V. Manca. P systems for biological dynamics. In [5], 81-126, 2006.

[3] L. Bianco, F. Fontana, and V. Manca. P systems with reaction maps. International Journal of Foundations of Computer Science, 17(1):27-48, February 2006.

[4] L. Bianco, D. Pescini, P. Siepmann, N. Krasnogor, F.J. Romero-Campero, and M. Gheorghe. Towards a P Systems Pseudomonas Quorum Sensing Model. Lecture Notes in Computer Science, 4361:197-214, 2007. 
[5] G. Ciobanu, M. J. Pérez-Jiménez, and G. Păun (Eds.). Applications of Membrane Computing (Natural Computing Series). Springer-Verlag New York, Inc., Secaucus, NJ, USA, 2006.

[6] F. Fontana and V. Manca. Discrete solution to differential equations by metabolic P systems. Theoretical Computer Science, 372:165-182, 2007.

[7] J. S. Huxley. Problems of Relative Growth. 2nd Ed., Dover, New York, 1972.

[8] D. S. Jones and B. D. Sleeman. Differential Equations and Mathematical Biology. Chapman \& Hall/CRC, February 2003.

[9] D. G. Luenberger. Optimization by Vector Space Methods, John Wiley \& Sons, Inc., 1969.

[10] V. Manca. Log-Gain Principles for Metabolic P Systems, In A. Condon et al. (Eds.), Algorithmic Bioprocesses, CHAPTER 28, Natural Computing Series, Springer-Verlag, Berlin Heidelberg, 2009.

[11] V. Manca. The Metabolic Algorithm for P systems: Principles and Applications. Theoretical Computer Science, 404:142-157, 2008.

[12] V. Manca. Fundamentals of metabolic P systems. In G. Păun, G. Rozenberg, and A. Salomaa, editors, Handbook of Membrane Computing, CHAPTER 16. Oxford University Press, 2009. To appear.

[13] V. Manca. Metabolic P dynamics. In G. Păun, G. Rozenberg, and A. Salomaa, editors, Handbook of Membrane Computing, CHAPTER 17. Oxford University Press, 2009. To appear.

[14] V. Manca and L. Bianco. Biological networks in metabolic P systems. BioSystems, 91(3):489-498, 2008.

[15] V. Manca, L. Bianco, and F. Fontana. Evolution and oscillation in P systems: Applications to biological phenomena. Lecture Notes in Computer Science, 3365:63-84, 2005.

[16] V. Manca, R. Pagliarini, and S. Zorzan. A photosynthetic process modelled by a metabolic P system. Natural Computing, 2009. DOI 10.1007/s11047-008-9104-x.

[17] G. Nicolis and I. Prigogine. Exploring Complexity. An Introduction. Freeman ans Company, San Francisco, CA, 1989.

[18] G. Păun. Membrane Computing: An Introduction. Springer, 2002.

[19] G. Păun and G. Rozenberg. A guide to membrane computing. Theoretical Computer Science, 287(1):73-100, September 2002.

[20] K. S. Scott. Chemical Chaos. Cambridge University Press, Cambridge, UK, 1991.

[21] A. M. Zhabotinsky. Proc. Acc. Sci, USRR. 157:392, 1964.

Roberto Pagliarini (July 23, 1981) earned his M. S. degree in Computer Science at University of Verona, where he is currently a PhD student in Computer Science. His research interests focus on System Biology, Molecular and Membrane Computing. He has collaborated with the biochemistry and vegetal physiology group at Biotechnological Department of Verona University, in order to investigate computational models for crucial events related to photosynthetic organisms. He is co-author of scientific papers on this subject. 
Giuditta Franco (August 5, 1974) graduated in Mathematics at the University of Pisa and earned her PhD in Computer Science, with a dissertation titled "Biomolecular Computing — Combinatorial Algorithms and Laboratory Experiments", at University of Verona, where she is currently an assistant professor. Her research interests focus on discrete mathematics and computational models of biological systems, namely on DNA and Membrane Computing. She gave talks in several international workshops and she is co-author of scientific papers published by prestigious sectoral journals. She is an effective member of both the European Molecular Computing Consortium (EMCC) and the International Society for Nanoscale Science, Computing and Engineering (ISNSCE).

Vincenzo Manca (March 9, 1949) is a Full Professor at the Computer Science Department of the University of Verona, where he is also Chair of the Bioinformatics programme. He obtained his degrees from the University of Pisa. His research interests cover a wide class of topics from mathematical logic, discrete mathematics, and theoretical computer science to informational analysis and computational models of biological systems. At present, his investigation is focused on "Natural Computing" (in particular DNA Computing and Membrane Computing, Synthetic Computational Biology). He is author of more than 100 scientific publications, appearing in international journals and scientific series. 